\title{
Increasing availability and consumption of single cigarettes: trends and implications for smoking cessation from the ITC Mexico Survey
}

\author{
Marissa G Hall, ${ }^{1}$ Nancy L Fleischer, ${ }^{2}$ Luz Myriam Reynales-Shigematsu, ${ }^{3}$ \\ Edna Arillo-Santillán, ${ }^{3}$ James $\mathrm{F}$ Thrasher ${ }^{3,4}$
}

'Department of Health Behavior, Gillings School of Global Public Health, University of North Carolina, Chapel Hill, North Carolina, USA

${ }^{2}$ Department of Epidemiology and Biostatistics, Arnold School of Public Health, University of South Carolina, Columbia, South Carolina, USA

${ }^{3}$ Tobacco Control Research Department, National Institute of Public Health Mexico, Cuernavaca, Mexico

${ }^{4}$ Department of Health Promotion, Education and Behavior, Arnold School of Public Health, University of South Carolina

\section{Correspondence to} Marissa G Hall, Department of Health Behavior, Gillings School of Global Public Health, University of North Carolina, Rosenau Hall, CB \#7440, Chapel Hill, NC 27599, USA; mghall@unc.edu

Received 24 March 2014 Accepted 19 August 2014 Published Online First 5 September 2014

\section{CrossMark}

\section{To cite: Hall MG,} Fleischer NL, ReynalesShigematsu LM, et al. Tob Control 2015;24:iii64-iii70.

\section{ABSTRACT}

Objective Determine (1) trends in single cigarette availability and purchasing in Mexico and (2) the association between neighbourhood access to singles and cessation behaviour among adult Mexican smokers. Methods We analysed data from Wave 4 (2010), Wave 5 (2011) and Wave 6 (2012) of the Mexican International Tobacco Control Policy Evaluation Survey. We used data from all three waves to examine time trends in singles availability and purchasing. To explore the association between neighbourhood access to singles and cessation behaviour, we used data from participants who were smokers at Wave 5 and followed up at Wave $6(n=1272)$.

Findings The percentage of participants who saw singles sold daily $(45.2 \%$ in $2010 ; 51.4 \%$ in 2011 ; $64.9 \%$ in 2012), who bought singles at least once a week $(22.3 \%$ in $2010 ; 29.1 \%$ in $2011 ; 29.1 \%$ in 2012$)$ and whose last cigarette purchase was a single (16.6\% in 2010; $20.7 \%$ in 2011; $25.8 \%$ in 2012) increased significantly from 2010 to 2012 (all $p<0.001$ ). The average percentage of residents who reported seeing singles sold daily in their neighbourhood in 2012 was $60 \%(S D=25 \%)$. In adjusted analyses, smokers living in neighbourhoods with higher access to singles were less likely to make a quit attempt (risk ratio $(\mathrm{RR})=0.72 ; 95 \%$ $\mathrm{Cl} 0.46$ to 1.12$)$, and more likely to relapse $(R R=1.30$; $\mathrm{Cl} 0.94$ to 1.82 ), but these results were not statistically significant.

Conclusions Single cigarettes appear widely accessible in Mexico and growing in availability. Future research should explore potential explanations, consequences and effective methods for reducing the availability of single cigarettes.

\section{INTRODUCTION}

Article 16 of the WHO Framework Convention on Tobacco Control (FCTC) calls for the prohibition of single cigarette sales. ${ }^{1}$ However, single cigarettes (also referred to as 'singles' in this article) are commonly sold and consumed in some low-income and middle-income countries (eg, India, Philippines, Bangladesh, Brazil, Thailand, Uruguay, Vietnam, Guatemala, Mexico) $)^{2-4}$ as well as in some urban, low-income neighbourhoods of high-income countries. ${ }^{5-7}$ Single cigarettes pose a potential threat to public health because they may be more affordable and accessible than packs or cartons for people with fewer resources, including minors. Two studies in Baltimore City, Maryland found that the most common reasons that smokers cited for buying single cigarettes were convenience and affordability. ${ }^{6} 7$ Moreover, the sale of singles may undermine the potential public health benefit of warning labels, as individuals purchasing single cigarettes are less likely to be exposed to warning labels on cigarette packaging.

In Mexico, about $16 \%$ of adults, or 11 million people, are current smokers, and more than $27 \%$ of adolescents aged 13-15 smoke cigarettes. ${ }^{8}$ The Mexican legal framework has included a ban of single cigarettes since 1999. In 2008, the General Law of Tobacco Control was passed in Mexico; Article 16, Section 1 of this law outlawed the distribution and sale of cigarettes in packages of fewer than 14 units. $^{9}$ Although single cigarettes have effectively been prohibited for 15 years, their sale appears prevalent in Mexico. The 2011 Mexican administration of the Global Youth Tobacco Survey, a representative survey of students aged 13-15 in 10 major cities, found that $50 \%$ of students had ever seen single cigarettes being sold, $21 \%$ had observed the sale of single cigarettes around their schools and 20\% had ever purchased single cigarettes. ${ }^{10}$ Data from the Mexican administration of the International Tobacco Control Policy Evaluation Survey (ITC) showed that, despite the long-standing ban on single cigarettes, in 2006, $9.1 \%$ of adult smokers bought singles at their last cigarette purchase. ${ }^{11}$

There are several potential explanations for the continued prevalence of singles. First, selling singles can be highly profitable for tobacco vendors; single cigarettes are generally sold for more than twice the per-unit price of packaged cigarettes. ${ }^{12}$ Second, there is confusion about which government agency is responsible for enforcing the ban. The Federal Commission for Protection against Health Risks is responsible for enforcing the ban at brick-and-mortar retailers, but the law does not specify which governing body is tasked with enforcing the ban among street vendors. This may in part explain why one 2011 study found that $91 \%$ of street vendors in Mexico sold single cigarettes, compared with $28 \%$ of brick-and-mortar retailers. ${ }^{13}$ Finally, corruption in Mexico may help to explain why the ban on singles has not been effectively enforced. According to Transparency International, 52\% of the Mexican population feels that government efforts to fight corruption are ineffective. ${ }^{14}$ A 2012 populationbased household survey of 1000 youth aged 13 and older found that corruption was cited as the most 
common reason why the ban on single cigarettes is not enforced, followed by authorities' lack of interest $(51 \%$ and $31 \%$, respectively). ${ }^{15}$

In the past several years, Mexico has implemented graphic warnings on cigarette packs, raised cigarette excise taxes and strengthened bans on smoking in workplaces, hospitality venues and some outdoor public spaces. ${ }^{11}{ }^{16}$ Examining trends in singles purchasing and availability may provide information about how smokers are responding to the stronger tobacco control environment in Mexico. Moreover, monitoring changes in the availability and purchasing of single cigarettes in Mexico over time can shed light on the extent to which Mexico has complied with Article 16 of the FCTC, and with the national law prohibiting the sale of single cigarettes. Thus, this study aims to assess changes in the purchasing and availability of single cigarettes among adult smokers in Mexico, over a period when diverse tobacco control policies were implemented (eg, taxes, pictorial warnings, smoke-free policies).

Research has shown that neighbourhood-level characteristics may influence individual-level smoking behaviour, even after controlling for individual characteristics. ${ }^{17-19}$ One neighbourhood characteristic that has not been studied in Mexico is the availability of single cigarettes at the neighbourhood level. Consensus is lacking about whether single cigarettes, on the whole, promote or inhibit cessation behaviour among adults. $^{20} 21$ Understanding the influence of neighbourhood access to single cigarettes on smoking behaviour is important for developing policies to address the tobacco epidemic, particularly in low-income and middle-income countries. To that end, a secondary aim of this study is to examine the correlates of neighbourhood-level access to single cigarettes, including the association between greater access and both quit attempts and smoking relapse among adult Mexican smokers.

\section{METHODS}

\section{Study sample}

The sample for the current study consisted of participants from Wave 4 (January to February 2010), Wave 5 (April to May 2011) and Wave 6 (October to November 2012) of the Mexican administration of the International Tobacco Control Policy Evaluation Survey (ITC Mexico). The survey included participants living in 150 census tracts, with an average of 15 participants per census tract in 2012 (range 4-22).

This study involves three analytic samples. The first sample, which included current smokers and former smokers from Waves 4 to $6(n=4249)$, was analysed to examine trends in singles purchasing and availability across all waves (ie, time trends sample). The second, a cross-sectional sample of all Wave $6(n=1971)$ smokers, was analysed to examine bivariate correlates of neighbourhood access to single cigarettes in 2012 (ie, neighbourhood correlates sample). This was performed because Wave 6 is the only survey wave for which the singles access question was asked with reference to the neighbourhood in which people lived. Finally, a longitudinal sample of participants, who were smokers at Wave 5 and followed up at Wave 6 $(n=1272)$, was used to analyse the association between neighbourhood access to single cigarettes and quit attempts and relapse (ie, quit behaviour sample). There was some loss to follow-up in the quit behaviour sample; $84 \%$ of participants from Wave 5 remained in the study at Wave 6. Those who dropped out did not differ from those who remained in the study on key characteristics, including sex, smoking intensity or how often they bought single cigarettes. However, those who dropped out of the study had lower quit intentions than those who remained in the study $(\mathrm{p}<0.05)$.

\section{Data collection}

ITC Mexico has collected data from a panel of adult smokers every $12-18$ months since $2006 .^{22}$ At each wave, the survey is replenished with new participants to make up for losses due to attrition and thereby maintain the sample size over time. Data collection for Waves 4-6 occurred in Mexico City, Tijuana, Guadalajara, Puebla, Mérida, Monterrey and León. We used a stratified, multistage sampling strategy, in which census tracts and then two block groups within each tract were selected with probability proportional to the number of households according to the 2005 Mexican national census. Households in each selected block group were in random order, and visited up to four times, in order to enumerate household members and recruit eligible smokers (ie, 18 years of age or older, smoked more than 100 cigarettes in their lifetime, and smoked at least once in the previous week). For each wave, sampling weights were developed to account for the likelihood of participant selection. These weights were then rescaled to the sample size at the city level for analytic efficiency in order to keep data from the largest cities from overwhelming data from smaller cities.

\section{Measures}

Primary study variables

Trend analysis: We used three measures to assess single cigarette purchasing and availability over time at the individual level. The first measure indicated whether smokers reported buying single cigarettes at least once a week. The second measure assessed whether the smokers' last purchase was a single cigarette. Finally, smokers were asked to report how often they saw single cigarettes sold anywhere, for which we dichotomised the response into daily versus all other responses.

Neighbourhood-level singles access analysis: Neighbourhood access to single cigarettes was defined as the proportion of residents in each census tract that reported seeing singles sold daily in their neighbourhood at Wave 6 (the reference to neighbourhood was not asked in prior waves). Responses from all Wave 6 participants were used to generate this variable, except for participants who had quit for more than 1 year $(n=2129)$.

Quit behaviour analysis: Quit attempts and relapse were measured at Wave 6, with quit attempts defined as reports of having tried to quit since the previous wave, including participants who indicated that they had quit at Wave 6. Smokers were considered to have relapsed if they made a quit attempt of any length between Waves 5 and 6, but reported that they were current smokers at Wave 6.

\section{Adjustment variables}

Quit intentions and smoking intensity: Quit intentions were defined as smokers intending to quit in the next 6 months. To measure smoking intensity, smokers were classified as non-daily, daily light ( $\leq 5$ cigarettes per day) and daily heavy ( $>5$ cigarettes per day), which represent tertile thresholds used in prior research in Mexico. ${ }^{23}$ Wave 5 data were used as baseline covariates in quit behaviour analyses.

Sociodemographic covariates: Analyses controlled for Wave 6 data on age, sex, highest educational level completed and monthly income. Education was classified as less than middle school; middle school; high school, vocational or incomplete university; and university or graduate school. Monthly income was coded as 0-3000 pesos, 3001-5000 pesos, 5001-8000 pesos, more than 8001 pesos and missing (at the time of data 
collection, US\$1 $\approx$ \$13 pesos). Neighbourhood deprivation was assessed using a composite measure based on the 2010 census data on education, health insurance, possession of goods and housing characteristics. ${ }^{24}$ On the basis of the median split, we dichotomised neighbourhood deprivation into very low and low versus medium, high or very high.

\section{Analysis}

We excluded cases with incomplete data on covariates of interest from analyses. All analyses were conducted using Stata V.12.1, except for generalised estimating equations (ie, neighbourhood correlates and quit behaviour analyses), which were run in SAS V.9.2.

\section{Trend data}

Using the time trends sample, we first examined trends in singles purchasing and availability across Waves 4-6. We then calculated the weighted percentage of participants in each wave who reported purchasing singles at least once a week, buying singles at their last purchase and seeing singles sold every day. We conducted $\chi^{2}$ tests to compare proportions across each adjacent wave using pairwise comparisons.

\section{Descriptive statistics}

We ran univariate descriptive statistics without weighting the data in order to characterise the ITC study sample. All other analyses accounted for the study design by using sampling weights.

\section{Correlates of neighbourhood access to singles}

For the regression modelling, we used generalised estimating equations, which correct for the within-neighbourhood nonindependence of observations when calculating the SEs of estimates. ${ }^{24}$ First, using the neighbourhood correlates sample, we examined bivariate correlates of neighbourhood access to single cigarettes, using data from the cross-sectional Wave 6 sample. This analysis aimed to identify variables that could potentially confound the relationship between neighbourhood access to singles and the outcomes of interest (quit attempts and relapse). We calculated the difference in mean percentages when examining the relationship between covariates and neighbourhood access to singles.

\section{Association of neighbourhood singles with quit attempts and relapse}

Next, using the quit behaviour sample, we examined the association between neighbourhood access to singles and quit attempts and relapse. In these analyses, we excluded participants who had quit for more than 1 year and participants who had quit smoking within the past 30 days at Wave 6 , based on recommendations that cessation be defined as quitting smoking for at least 4 weeks. ${ }^{25} 26$ For these outcomes, we used logbinomial models for estimating risk ratios, the recommended approach for modelling binary outcomes with a prevalence greater than $10 \% .^{27}$ In the first set of models, we examined the bivariate association between neighbourhood access to singles and both outcomes. The second model for quit attempts adjusted for sex, education, income, quit intentions and smoking intensity. The second model for relapse adjusted for sex, education, quit intentions and smoking intensity. Income was not significantly correlated with neighbourhood access to singles in bivariate analyses, so we excluded income from the second relapse model to allow the model to converge in SAS. In order for the models to converge, we also excluded age from the quit attempts and relapse models; age was not significantly associated with quit attempts or relapse in bivariate regression. The third set of models adjusted for neighbourhood deprivation in addition to the individual-level covariates included in the second set of models.

\section{RESULTS}

We explored trends in singles purchasing and availability over time, using data from Waves 4 to 6 of the ITC survey (figure 1). The percentage of people who saw singles sold daily anywhere increased from Wave $4(45.2 \%$, CI $40.6 \%$ to $49.8 \%)$ to Wave 5 (51.4\%, CI $46.1 \%$ to $56.6 \% ; \mathrm{p}<0.001)$, and again from Wave 5 to Wave $6(64.9 \%$, CI $60.4 \%$ to $69.5 \% ; \mathrm{p}<0.001)$. The percentage of smokers who bought singles at least once a week increased from Wave $4(22.3 \%$, CI $19.1 \%$ to $25.4 \%)$ to Wave 5 (29.1\%, CI $24.6 \%$ to $33.6 \%$; $<<0.001)$, but did not increase from Wave 5 to Wave $6(29.1 \%$, CI $24.1 \%$ to $34.1 \%$; $\mathrm{p}=0.998$ ). Finally, the percentage of smokers whose last cigarette purchase was a single cigarette increased from Wave 4 $(16.6 \%$, CI $13.9 \%$ to $19.3 \%)$ to Wave $5(20.7 \%$, CI $16.7 \%$ to $24.6 \% ; \mathrm{p}=0.002)$, and again from Wave 5 to Wave $6(25.8 \%$, CI $21.9 \%$ to $29.7 \%$; $<0.001$ ).

We present descriptive data for the Wave 6 sample and the quit behaviour sample in table 1 . Within both of these samples, about two-thirds of participants were male, and the mean age was approximately 43 years. Two-thirds of participants had completed less than a high school education, one-quarter had completed vocational, high school or some university and 10\% had completed university or graduate school. More than half of the participants reported buying singles at least a few times in the past 6 months. Most participants (58\%) reported seeing singles sold in their neighbourhood every day in Wave 6 . The average neighbourhood-level percentage of residents who reported seeing singles sold in their neighbourhood every day was $60 \%(\mathrm{SD}=30 \%)$ in Wave 6 . The correlation between education and income was low, as was the correlation between neighbourhood deprivation and both education and income (data not shown). Within the quit behaviour sample, 34\% had made a quit attempt since the last wave. Of those who made a quit attempt, 26\% successfully quit and 74\% relapsed.

We assessed the bivariate relationships between the neighbourhood-level percentage of smokers who reported seeing singles sold in their neighbourhood every day and neighbourhood deprivation, individual-level demographic variables, smoking intensity and smoking status (data not shown). None

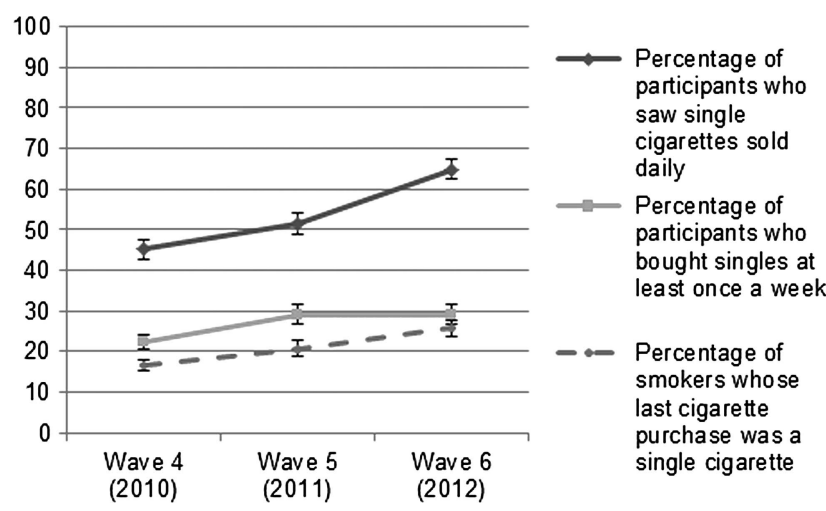

Figure 1 Trends in singles availability and purchasing in Mexico from 2010 to 2012, time trends sample. Graph depicts SE bars for point estimates. Weighted data used. 
Table 1 Sample characteristics of adult Mexican smokers from the International Tobacco Control Policy Evaluation Survey (ITC) survey, 2011-2012

\begin{tabular}{|c|c|c|}
\hline & $\begin{array}{l}\text { Neighbourhood } \\
\text { correlates sample (Wave } \\
\text { 6) } n(\%)\end{array}$ & $\begin{array}{l}\text { Quit behaviour } \\
\text { sample (Waves } 5 \\
\text { and 6) n (\%) }\end{array}$ \\
\hline $\mathrm{N}$ & 1971 & 1272 \\
\hline \multicolumn{3}{|l|}{ Demographics } \\
\hline \multicolumn{3}{|l|}{ Sex } \\
\hline Male & $1230(62.4)$ & $796(62.6)$ \\
\hline \multicolumn{3}{|l|}{ Age } \\
\hline Mean, years (SD) & $43.4(15.3)$ & $43.7(14.9)$ \\
\hline \multicolumn{3}{|l|}{ Education } \\
\hline$<$ Middle school & $609(30.9)$ & $403(31.7)$ \\
\hline Middle school & $645(32.7)$ & $428(33.7)$ \\
\hline $\begin{array}{l}\text { Vocational, high school, } \\
\text { incomplete university }\end{array}$ & $522(26.5)$ & $311(24.5)$ \\
\hline $\begin{array}{l}\text { University or graduate } \\
\text { school }\end{array}$ & $195(9.9)$ & $130(10.2)$ \\
\hline \multicolumn{3}{|l|}{ Income (pesos/month) } \\
\hline $0-3000$ & $496(25.2)$ & $319(25.1)$ \\
\hline $3001-5000$ & $640(32.5)$ & $427(33.6)$ \\
\hline $5001-8000$ & $394(20.0)$ & $255(20.1)$ \\
\hline$>8001$ & $268(13.6)$ & $161(12.7)$ \\
\hline Missing & $173(8.8)$ & $110(8.7)$ \\
\hline \multicolumn{3}{|l|}{ Smoking behaviour } \\
\hline \multicolumn{3}{|l|}{ Smoking status } \\
\hline Quitter & $325(16.5)$ & $112(8.8)$ \\
\hline Smoker & $1646(83.5)$ & $1160(91.2)$ \\
\hline \multicolumn{3}{|l|}{ How often bought singles* } \\
\hline Daily & & $133(10.5)$ \\
\hline $\begin{array}{l}\text { Not daily but once a week } \\
\text { or more }\end{array}$ & & $266(20.9)$ \\
\hline One to three times a month & & $137(10.8)$ \\
\hline $\begin{array}{l}\text { A few times in the past } \\
6 \text { months }\end{array}$ & & $183(14.4)$ \\
\hline Never in the past 6 months & & $553(43.5)$ \\
\hline \multicolumn{3}{|l|}{ Smoking intensity* } \\
\hline Non-daily & & $393(30.9)$ \\
\hline Daily $\leq 5$ cigarettes/day & & $402(31.6)$ \\
\hline Daily $>5$ cigarettes/day & & $477(37.5)$ \\
\hline \multicolumn{3}{|l|}{ Quitting behaviour } \\
\hline \multicolumn{3}{|c|}{ Quit intention in the next 6 months* } \\
\hline No & & $1077(84.7)$ \\
\hline Yes & & $195(15.3)$ \\
\hline \multicolumn{3}{|l|}{ Tried to quit since the last wave } \\
\hline No & & $859(67.5)$ \\
\hline Yes & & $413(32.5)$ \\
\hline Relapsed since the last wave & & $n=409$ \\
\hline No & & $108(26.4)$ \\
\hline Yes & & $301(73.6)$ \\
\hline \multicolumn{3}{|l|}{ Neighbourhood characteristics } \\
\hline \multicolumn{3}{|c|}{ Percentage of residents who report seeing singles sold in the neighbourhood every day } \\
\hline Mean (SD) & $60 \%(30 \%)$ & $60 \%(20 \%)$ \\
\hline \multicolumn{3}{|l|}{ Neighbourhood deprivation } \\
\hline Very low or low & $892(45.3)$ & $569(44.7)$ \\
\hline Medium, high or very high & 1079 (54.7) & $703(55.3)$ \\
\hline
\end{tabular}

of the individual-level variables were significantly associated with neighbourhood access to singles. Those living in neighbourhoods with medium, high or very high levels of deprivation had higher neighbourhood access to singles than those living in neighbourhoods with low or very low levels of deprivation (difference in mean $=10.4 \%$, CI $1.4 \%$ to $19.3 \%$ ).

Higher neighbourhood access to singles was associated with lower likelihood of quit attempts after controlling for covariates; however, the CI was wide and the relationship was not statistically significant (risk ratio $(\mathrm{RR})=0.72$, CI 0.46 to 1.12 ; table 2 , model 3). In adjusted analyses, people with monthly incomes of 3001-5000 pesos (US\$230-US\$384) were more likely to make a quit attempt than those with incomes less than 3000 pesos (less than US\$230; RR $=1.40$, CI 1.07 to 1.83 ), as were those with incomes of 5001-8000 pesos (US\$384-US\$614; $\mathrm{RR}=1.43$, CI 1.03 to 1.99 ; table 2, model 2). Quit intentions were significantly associated with making a quit attempt $(\mathrm{RR}=1.53$, CI 1.27 to 1.83$)$. Smoking intensity was also associated with quit attempts; daily smokers who smoked five or fewer cigarettes per day were less likely than non-daily smokers to make a quit attempt $(\mathrm{RR}=0.59$, CI 0.47 to 0.75$)$, as were smokers who smoked six or more cigarettes per day $(R R=0.59$, CI 0.46 to 0.74 ). Neighbourhood deprivation was not significantly associated with quit attempts (table 2, model 3).

After controlling for individual covariates, higher neighbourhood access to singles was associated with a higher probability of relapse; however, the CI was wide and the relationship was not statistically significant $(\mathrm{RR}=1.31$, CI 0.94 to 1.81 ; table 3, model 2). Participants who had a vocational, high school or incomplete university education were 1.22 times as likely to relapse as those with less than a middle school education (CI 1.00 to 1.48$)$. Neighbourhood deprivation was not associated with relapse, and the effect of neighbourhood access to singles remained virtually unchanged after controlling for neighbourhood deprivation ( $\mathrm{RR}=1.30$, CI 0.94 to 1.82 ; table 3, model 3).

\section{DISCUSSION}

This study found that the proportion of adult smokers in Mexico who bought singles increased from 2010 to 2012, whether we examined the last purchase or purchase at least once a week. The proportion of smokers who saw singles sold daily also increased over this time, with almost $60 \%$ of smokers reporting that they saw singles sold in their neighbourhood every day in 2012. Hence, single cigarettes appear widely accessible and growing in their availability. A similar trend was observed in an environmental assessment study, which monitors the point-of-sale environments in brick-and-mortar retailers and among street vendors in Mexico. This study found that in Mexico City, the sale of single cigarettes increased from 2008 to 2011 among brick-and-mortar tobacco retailers (from $4.6 \%$ to $27.8 \%$ ) and street vendors (from $41.9 \%$ to $90.9 \%)^{13}$

Our study did not examine the reasons why singles purchasing and availability have increased over time. However, one potential explanation could be smokers' responses to a stronger tobacco control environment. Evidence from the USA suggests that tobacco control policies may lead to an increase in light smoking. ${ }^{28} 29$ Mexican smokers tend to smoke fewer cigarettes per day than smokers in other countries, including the USA. ${ }^{23}$ There is some evidence that light smoking has become more prevalent over time in Mexico. Data from the 2000 National Health Survey and the 2006 and 2012 National Surveys of Health and Nutrition indicate that the average number of cigarettes consumed per day among Mexican adult smokers decreased significantly over time, from 8.2 cigarettes per day in 2000 , to 7.5 cigarettes per day in 2006 , to 6.3 cigarettes per day in 2012. ${ }^{30}$ The upcoming 2014 Mexican administration of the Global Adult Tobacco Survey (GATS) will provide additional information on whether per capita cigarette consumption has 
declined in Mexico since the first GATS survey in 2009. Changes in cigarette consumption in Mexico may be due to a stronger tobacco control environment. Mexico has recently implemented several policies that make smoking less socially acceptable and less desirable. For example, in 2008, Mexico banned smoking in some indoor workplaces and public places, ${ }^{31}$ and in 2010 it began requiring graphic warning labels on cigarette packs. ${ }^{32} 33$ These policies may make smoking less socially acceptable, ${ }^{34}$ causing smokers to purchase and smoke single cigarettes more irregularly and to avoid carrying cigarette packages with a prominent package warning. As such, these policies may help undermine the attractiveness of the branded package. Moreover, a series of tax increases have been implemented in Mexico since 2007 , including a substantial tax in 2011, decreasing consumption. ${ }^{1135}$ In the end, however, it is unclear whether the apparently greater availability of singles is mainly due to decreased consumer demand or to vendor strategies to increase profits, perhaps in the face of decreasing demand. Future research should examine other potential explanations for the increase in the availability and purchasing of single cigarettes.

This paper also examined the influence of neighbourhood access to singles-a potentially important neighbourhood-level predictor of smoking behaviour-on quit attempts and relapse. In adjusted analyses, we did not find a significant impact, although the point estimates suggest that greater neighbourhood access to singles may be associated with a lower probability of making a quit attempt and a higher probability of relapse. The lack of an association could signify that neighbourhood access to single cigarettes simply does not have an impact on quit attempts or relapse. Lack of statistical power could have also contributed to the null findings. Many of our estimates have wide CIs due to a limited sample size at the neighbourhood level $(n=150)$, which limits the power in nested studies. However, another potential explanation is that competing mechanisms are at play: some smokers may use singles as a method for trying to cut down and to eventually quit, whereas others may be cued to smoke by seeing singles being sold. ${ }^{20} 21$ Data from the 2008 ITC Mexico Survey showed that 24\% of adult smokers reported using single cigarettes as a strategy for reducing cigarette consumption. ${ }^{20}$ Although single cigarettes have lower upfront costs than packs, the per-unit cost of a single cigarette is approximately double the cost when buying by the pack. A qualitative study with Mexican smokers revealed that some smokers controlled their consumption by imposing the additional financial cost of buying singles, as well as the logistical effort of buying a single cigarette each time they wanted to smoke. $^{21}$

On the other hand, some of the impact of using single cigarettes as a harm reduction tactic may be partially offset by smoking urges that are triggered by exposure to single cigarettes. Data from the 2008 ITC Mexico Survey demonstrate that $40 \%$ of smokers reported cravings to smoke after seeing the sale of single cigarettes, and the frequency of cravings to smoke after seeing singles being sold was positively associated with single cigarette consumption. ${ }^{20}$ Smokers who reported more frequent urges to smoke after viewing singles were more likely to buy singles, but they were no less likely to quit than those who did not report these same urges. $^{20}$ Similarly,

Table 2 Risk ratios for quit attempts associated with sociodemographic and smoking characteristics, quit behaviour sample, Mexico 2011$2012(n=1272)$

\begin{tabular}{|c|c|c|c|}
\hline & \multicolumn{3}{|c|}{ prevalence ratio $(95 \% \mathrm{Cl})$} \\
\hline & Model 1 & Model 2 & Model 3 \\
\hline Neighbourhood access to singles & $0.79(0.45$ to 1.40$)$ & $0.72(0.47$ to 1.09$)$ & 0.72 (0.46 to 1.12 ) \\
\hline \multicolumn{4}{|l|}{ Sex } \\
\hline Female & & 1 & 1 \\
\hline Male & & 0.83 (0.71 to 0.99$)$ & 0.84 (0.71 to 0.99$)$ \\
\hline \multicolumn{4}{|l|}{ Education } \\
\hline$<$ Middle school & & 1 & 1 \\
\hline Middle school & & 0.90 (0.73 to 1.11$)$ & $0.90(0.73$ to 1.11$)$ \\
\hline Vocational, high school, incomplete university & & 0.98 (0.77 to 1.25$)$ & $0.98(0.77$ to 1.25$)$ \\
\hline University or graduate school & & 1.05 (0.79 to 1.40$)$ & $1.05(0.79$ to 1.40$)$ \\
\hline \multicolumn{4}{|l|}{ Income } \\
\hline $0-3000$ & & 1 & 1 \\
\hline $3001-5000$ & & 1.40 (1.07 to 1.83$)$ & 1.40 (1.07 to 1.83$)$ \\
\hline $5001-8000$ & & 1.43 (1.03 to 1.99$)$ & $1.43(1.03$ to 1.99$)$ \\
\hline$>8001$ & & 1.25 (0.89 to 1.76$)$ & 1.25 (0.89 to 1.76$)$ \\
\hline Missing & & 1.20 (0.82 to 1.76$)$ & 1.20 (0.82 to 1.76$)$ \\
\hline \multicolumn{4}{|l|}{ Quit intention in the next 6 months } \\
\hline No & & 1 & 1 \\
\hline Yes & & 1.53 (1.27 to 1.84$)$ & $1.53(1.27$ to 1.84$)$ \\
\hline \multicolumn{4}{|l|}{ Smoking intensity } \\
\hline Non-daily & & 1 & 1 \\
\hline Daily $\leq 5$ cigarettes/day & & 0.59 (0.47 to 0.75$)$ & $0.59(0.47$ to 0.75$)$ \\
\hline Daily $>5$ cigarettes/day & & 0.59 (0.46 to 0.74$)$ & 0.59 (0.46 to 0.74$)$ \\
\hline \multicolumn{4}{|l|}{ Neighbourhood deprivation } \\
\hline Very low or low & & & 1 \\
\hline Medium, high or very high & & & 0.98 (0.80 to 1.22$)$ \\
\hline
\end{tabular}

Age was excluded to allow models to converge. Weighted data used. 
Table 3 Risk ratios for smoking relapse associated with sociodemographic and smoking characteristics, quit behaviour sample, Mexico 2011-2012 $(n=409)$

\begin{tabular}{|c|c|c|c|}
\hline & \multicolumn{3}{|c|}{ prevalence ratio $(95 \% \mathrm{Cl})$} \\
\hline & Model 1 & Model 2 & Model 3 \\
\hline Neighbourhood access to singles & $1.28(0.92$ to 1.77$)$ & $1.31(0.94$ to 1.81$)$ & $1.30(0.94$ to 1.82$)$ \\
\hline \multicolumn{4}{|l|}{ Sex } \\
\hline Female & & 1 & 1 \\
\hline Male & & $1.12(0.97$ to 1.28$)$ & 1.12 (0.97 to 1.28$)$ \\
\hline \multicolumn{4}{|l|}{ Education } \\
\hline$<$ Middle school & & 1 & 1 \\
\hline Middle school & & $1.15(0.95$ to 1.39$)$ & $1.15(0.95$ to 1.40$)$ \\
\hline Vocational, high school, incomplete university & & $1.22(1.00$ to 1.48$)$ & 1.22 (1.00 to 1.48$)$ \\
\hline University or graduate school & & $1.07(0.79$ to 1.44$)$ & 1.07 (0.79 to 1.45$)$ \\
\hline \multicolumn{4}{|l|}{ Quit intention in the next 6 months } \\
\hline No & & 1 & 1 \\
\hline Yes & & $0.97(0.83$ to 1.14$)$ & 0.97 (0.83 to 1.14$)$ \\
\hline \multicolumn{4}{|l|}{ Smoking intensity } \\
\hline Non-daily & & 1 & 1 \\
\hline Daily $\leq 5$ cigarettes/day & & $1.02(0.85$ to 1.22$)$ & $1.02(0.85$ to 1.22$)$ \\
\hline Daily $>5$ cigarettes/day & & $1.02(0.87$ to 1.20$)$ & $1.02(0.87$ to 1.20$)$ \\
\hline \multicolumn{4}{|l|}{ Neighbourhood deprivation } \\
\hline Very low or low & & & 1 \\
\hline Medium, high or very high & & & $1.00(0.88$ to 1.14$)$ \\
\hline
\end{tabular}

smokers who purchased singles to control their consumption were not more likely to attempt to quit than those who did not. ${ }^{20}$ Future research should examine whether using singles as a quit strategy has an effect on cessation behaviour. Additional studies with smokers should also consider the role of single cigarettes in unassisted quit attempts (ie, quitting cold turkey or reducing consumption before quitting), as research shows that most former smokers quit without any form of assistance. $^{36}$ Finally, future research should examine the influence of other characteristics of the retail environment on smoking behaviour, including urges to smoke when seeing packages of cigarettes, which are often sold by street vendors alongside singles and that are more prominently displayed in brick-and-mortar points of sale. ${ }^{13}$

\section{STRENGTHS AND LIMITATIONS}

This study used a probability-based sampling approach, which improves the representativeness and generalisability of our findings. Our study also benefits from having a longitudinal assessment of cessation behaviour. However, our findings may not generalise to rural populations or to Mexicans living in cities that were not included in the sample. Nevertheless, three-quarters of Mexicans live in urban areas, and the cities where we collected data are among the most populated in the country. ${ }^{37}$ Our measure of neighbourhood access to singles relied on self-report, and therefore could be influenced by respondents' purchasing decisions and perceptions of availability. However, our novel measurement approach uses individual responses to create a composite measure of singles availability for neighbourhoods, which may limit the likelihood that our exposure measure is as biased as exposure that relies solely on the perception of the individual for whom future behaviour is predicted. Future research should involve the collection of objective measures of neighbourhood access to singles. The percentage of smokers who bought singles at their last purchase showed a similar time trend but slightly different point estimates than was found in a previous study that used ITC Mexico data. ${ }^{11}$ These estimates differ because we used a slightly larger analytic sample of ITC Mexico Survey participants and because we used wave-specific, non-rescaled weights to derive prevalence estimates. Although we adjusted for several individual and neighbourhood covariates, factors not included in our models (such as access to medications, working and living in a smoke-free home) could confound the relationship between neighbourhood access to singles and the outcomes of interest. Finally, the small sample size, particularly for the relapse sample, reduced our statistical power.

\section{CONCLUSIONS}

Our study demonstrates that single cigarettes are commonly purchased and sold in Mexico and that singles purchasing and availability have increased since 2010. Mexico's long-standing ban of single cigarette sales appears to be largely ignored by vendors and enforcement agencies. Our findings point towards the need for better enforcement strategies of the ban on single cigarettes, such as a clear definition of the entities responsible for enforcement, licensing of tobacco retailers and frequent compliance checks. Future research should explore how tobacco control policies-particularly in low-income and middle-income countries-influence single cigarette consumption and, in turn, cessation behaviour.

\section{What this paper adds}

- Despite a long-standing ban on single cigarette sales in Mexico, single cigarettes were widely available and frequently purchased among adult Mexican smokers.

- Trend data indicate that the availability and purchasing of single cigarettes increased from 2010 to 2012 in Mexico. 
Acknowledgements Authors thank the research participants for taking part in this study. They also thank Rosaura Pérez Hernandez for her help with data collection and analysis.

Contributors MGH formulated the research, conducted statistical analyses and drafted the paper. NLF formulated the research, provided extensive analytic support and edited multiple drafts of the paper. JFT formulated the research, oversaw data collection, assisted with data analysis and edited multiple drafts of the paper. LMRS and EAS reviewed and edited the paper several times.

Funding Funding for data collection from Waves 4 and 5 came from the Mexican Consejo Nacional de Ciencia y Tecnología (Salud-2007-C01-70032), and Wave 6 data were collected with funding from a grant from the Canadian Institutes of Health Research (115016). The analysis was partially supported by a grant from the National Cancer Institute at the National Institutes of Health (P01 CA138389), as well as the 2012 Latin America/Latino Health Summer Fellowship at the University of North Carolina. Additional support was provided to Geoffrey T. Fong from a Senior Investigator Award from the Ontario Institute for Cancer Research and a Prevention Scientist Award from the Canadian Cancer Society Research Institute.

Competing interests None declared.

Ethics approval The ITC Mexico Survey was cleared for ethics by Research Ethics Boards or International Review Boards at the Instituto Nacional de Salud Pública (Mexico) and the University of Waterloo (Canada). The ITC Mexico Survey was cleared for ethics by Research Ethics Boards or International Review Boards at the Instituto Nacional de Salud Pública (Mexico) and the University of Waterloo (Canada).

Provenance and peer review Not commissioned; externally peer reviewed.

\section{REFERENCES}

1 World Health Organization. Framework Convention on Tobacco Control. 2013.

2 de Ojeda A, Barnoya J, Thrasher JF. Availability and costs of single cigarettes in Guatemala. Nicotine Tob Res 2013;15:83-7.

3 Elf JL, Modi B, Stillman F, et al. Tobacco sales and marketing within 100 yards of schools in Ahmedabad city, India. Public Health 2013;127:442-8.

4 Kostova D, Chaloupka FJ, Yurekli A, et al. A cross-country study of cigarette prices and affordability: evidence from the Global Adult Tobacco Survey. Tob Control 2014;23:e3.

5 Latkin CA, Murray LI, Clegg Smith K, et al. The prevalence and correlates of single cigarette selling among urban disadvantaged drug users in Baltimore, Maryland. Drug Alcohol Depend 2013;132:466-70.

6 Stillman FA, Bone L, Avila-Tang E, et al. Barriers to smoking cessation in inner-city African American young adults. Am J Public Health 2007;97:1405-8.

7 Stillman FA, Bone LR, Milam AJ, et al. Out of view but in plain sight: the illegal sale of single cigarettes. J Urban Health 2014;91:355-65.

8 Campaign for Tobacco Free Kids (2011). Mexico: Tobacco burden facts. http://global. tobaccofreekids.org/files/pdfs/en/Mexico_tob_burden_en.pdf (accessed 1 Apr 2013).

9 Diario Oficial de la Federación. Ley general para el control de tabaco. 2008.

10 Reynales-Shigematsu LM, Rodríguez-Bolaños R, Ortega-Ceballos P, et al. Encuesta de tabaquismo en jóvenes. Mexico: Instituto Nacional de Salud Pública, 2011.

11 de Miera Juárez BS, Thrasher JF, Shigematsu LMR, et al. Tax, price and cigarette brand preferences: a longitudinal study of adult smokers from the ITC Mexico Survey. Tob Control 2013;23(Suppl 1):i80-5.

12 Reyes D. Como "pan caliente" se venden cigarros sueltos. Diario Puntual 2012.

13 Rodríguez Bolaños R, Reynales Shigematsu LM, Barrientos Gutierrez T, et al. Vigilancia y monitoreo de las estrategias para el control del tabaco: un análisis a través del Sistema de Información Geográfica. In: Reynales Shigematsu LM, Thrasher JF, Lazcano $\mathrm{E}_{1}$, et al, eds. Política pública para el control del tabaco en México. Cuernavaca, Mexico: Instituto Nacional de Salud Pública, 2013:84-96.

14 Transparency International. Corruption perceptions index 2012. 2012.

15 Consejo Nacional Contra el Tabaquismo. Estudio nacional de tabaquismo: Encuesta en vivienda. 2012.

16 Campaign for Tobacco Free Kids. Country details for Mexico. 2013.

17 Diez Roux AV, Merkin SS, Hannan P, et al. Area characteristics, individual-level socioeconomic indicators, and smoking in young adults: the coronary artery disease risk development in young adults study. Am J Epidemiol 2003:157:315-26.

18 Pearce J, Hiscock R, Moon G, et al. The neighbourhood effects of geographical access to tobacco retailers on individual smoking behaviour. J Epidemiol Community Health 2009;63:69-77.

19 Sundquist J, Malmstrom M, Johansson SE. Cardiovascular risk factors and the neighbourhood environment: a multilevel analysis. Int I Epidemiol 1999:28:841-5.

20 Thrasher JF, Villalobos V, Barnoya J, et al. Consumption of single cigarettes and quitting behavior: a longitudinal analysis of Mexican smokers. BMC Public Health 2011:11:134.

21 Thrasher JF, Villalobos V, Dorantes-Alonso A, et al. Does the availability of single cigarettes promote or inhibit cigarette consumption? Perceptions, prevalence and correlates of single cigarette use among adult Mexican smokers. Tob Control 2009;18:431-7.

22 Fong GT, Cummings KM, Borland R, et al. The conceptual framework of the International Tobacco Control (ITC) Policy Evaluation Project. Tob Control 2006;15 (Suppl 3):iii3-11.

23 Swayampakala K, Thrasher J, Carpenter MJ, et al. Level of cigarette consumption and quit behavior in a population of low-intensity smokers-longitudinal results from the International Tobacco Control (ITC) survey in Mexico. Addict Behav 2013;38:1958-65.

24 Liang K-Y, Zeger SL. Longitudinal data analysis using generalized linear models. Biometrika 1986;73:13-22.

25 Hughes JR, Keely JP, Niaura RS, et al. Measures of abstinence in clinical trials: issues and recommendations. Nicotine Tob Res 2003;5:13-25.

26 IARC. Handbooks of cancer prevention. Tobacco control: methods for evaluating tobacco control policies. Lyon, France: International Agency for Research on Cancer, 2009.

27 Deddens JA, Petersen MR. Approaches for estimating prevalence ratios. Occup Environ Med 2008;65:481, 501-6.

28 Gilpin EA, Pierce JP. The California Tobacco Control Program and potential harm reduction through reduced cigarette consumption in continuing smokers. Nicotine Tob Res 2002;4(Suppl 2):S157-66.

29 Pierce JP, White MM, Messer K. Changing age-specific patterns of cigarette consumption in the United States, 1992-2002: association with smoke-free homes and state-level tobacco control activity. Nicotine Tob Res 2009;11:171-7.

30 Guerrero-Lopez CM, Munos-Hernandez JA, Saenz de Miera-Juarez B, et al. [Tobacco consumption, mortality and fiscal policy in Mexico]. Salud Publica Mex 2013;55 (Suppl 2):S276-81.

31 Thrasher JF, Boado M, Sebrié EM, et al. Smoke-free policies and the social acceptability of smoking in Uruguay and Mexico: findings from the International Tobacco Control Policy Evaluation Project. Nicotine Tob Res 2009;11:591-9.

32 Thrasher JF, Murukutla N, Perez-Hernandez R, et al. Linking mass media campaigns to pictorial warning labels on cigarette packages: a cross-sectional study to evaluate effects among Mexican smokers. Tob Control 2013;22(e1): e57-65.

33 Thrasher JF, Perez-Hernandez R, Arillo-Santillan E, et al. [Towards informed tobacco consumption in Mexico: effect of pictorial warning labels in smokers]. Salud Publica Mex 2012;54:242-53.

34 Thrasher JF, Pérez-Hernández R, Swayampakala K, et al. Policy support, norms, and secondhand smoke exposure before and after implementation of a comprehensive smoke-free law in Mexico City. Am J Public Health 2010;100:1789-98.

35 Saenz-de-Miera B, Thrasher JF, Chaloupka FJ, et al. Self-reported price of cigarettes, consumption and compensatory behaviours in a cohort of Mexican smokers before and after a cigarette tax increase. Tob Control 2010;19:481-7.

36 Chapman S, MacKenzie R. The global research neglect of unassisted smoking cessation: causes and consequences. PLoS Med 2010;7:e1000216.

37 Consejo Nacional de Población. Delimitación de las zonas metropolitanas de México 2010. Mexico City, Mexico: Secretaría de Desarrollo Social, Consejo Nacional de Población, Instituto Nacional de Estadística y Geografía, 2012. 